\title{
PROSPECTIVE IMPROVEMENTS FOR SAFER FUEL TANKS: EXPERIMENTAL TESTS
}

\author{
G. JANSZEN, A. GALBIATI \& L. DI LANDRO \\ Department of Aerospace Science and Technologies, Politecnico di Milano, Italy.
}

\begin{abstract}
In past studies, some of the authors presented how the integration of different systems, for the prevention of fires or explosions due to impact or bullet damage, may significantly improve the safety of fuel tanks. Leakage, after bullet penetration or debris impact, can be significantly reduced by introducing polymeric materials with self-healing capabilities for the container's walls, while an internal aluminium filler can reduce the sloshing and the danger of fuel ignition. In the present paper, an experimental evaluation of the proposed solution is presented. A ballistic test campaign on a fluid container was performed to investigate the interaction between an ethylene-methacrylic acid (EMAA)-based ionomeric wall (i.e. Dupont ${ }^{\circledR}$ Surlyn 8940) and an internal aluminium filler (i.e. Explosafe ${ }^{\circledR}$ ). Results show that the presence of the fluid increases the self-healing capabilities, which are however slightly affected by the internal aluminium filler; the contribution in terms of sloshing reduction remains relevant. Moreover, additional configurations based on multilayer panels are presented. The authors studied the healing process of EMAA in a sandwich configuration made of one skin of ionomer and one skin of carbon fibre, separated by an aramidic honeycomb. The main objective of the honeycomb is to prevent the remarkable reduction of the healing capabilities observed when ionomer is directly coupled to aramidic fabric or composite panels. The new multilayer configurations have been tested at different impact conditions.

Keywords: fillers, fuel tanks, impact, self-healing.
\end{abstract}

\section{INTRODUCTION}

Fuel tanks integrity is one of the most concerns and specifically designed configuration, and materials may significantly improve safety margins in case of bullet perforation or debris impact. In such situations, deflagration can be activated by sudden variation in internal pressure and temperature or liquid spilling due to wall container perforation [1]. Cellular filler material in the container can remarkably dispel fire heat and limit pressure wave peaks generated by a bullet explosion; moreover, the overall response to impact loads in case of a crash is remarkably improved [2,3].

In traditional multilayer wall, fuel tanks self-sealing capability can be obtained as a result of swelling or chemical reaction of rubber/foam layer when it comes in contact with the spilling internal liquid. Thermoplastic polymers, such as ethylene-co-methacrylic acid (EMAA) copolymers and ionomers have shown a self-healing behaviour after ballistic impacts either in low and hypervelocity speed range [4,5]. This distinctive feature has been studied, demonstrating how the repair of damages after an impact event is autonomous and instantaneous without any external intervention.

On the other hand, despite their self-healing capacity, these materials cannot be used in primary structural components due to their low mechanical properties. A possible solution to this issue can be the use of EMAA ionomers in multilayer composite structures to add the self-healing functionality after high-energy impact to the structural strength and stiffness.

This paper is part of the Proceedings of the $6^{\text {th }}$ International Conference on

Safety and Security Engineering (SAFE 2015)

www.witconferences.com 
Multilayer configurations made of EMAA ionomer with carbon foam or aramidic fabric interposed have been studied in previous works. Results of these works showed a general good self-healing behaviour, although the presence of the reinforcing composite or foam efficiently adherent to ionomer in some cases reduced the autonomic mending efficiency. This was mainly due to the deformation constraints provided by the foam or the fabric, which impaired the healing ability of the material [6,7]. A possible solution is the use of a honeycomb core interposed between the ionomer and the composite panel. The lower stiffness of the core compared to that of the reinforcing panel will allow the ionomer to deform freely, not preventing hole closure after projectile passage.

To evaluate the new fuel tank concept in which the walls are made of rigid self-healing polymer in a multilayer composite, different ballistic tests have been carried out. The research proceeded in two separate ways. A multilayer standalone test campaign was carried out at low impact speeds with different bullet diameters versus sample thickness ratios. Impact angle was varied too.

At the same time, the effect of the fluid presence on the ionomer self-healing behaviour was evaluated by testing a water filled tank mock-up with ionomer walls. Similar tests have been conducted with the tank containing an anti-sloshing filler material (Explosafe ${ }^{\circledR}$ ) with the aim to evidence possible effects of internal pressure variation over the self-mending capacity.

\section{EXPERIMENTAL}

\subsection{Materials and samples production}

In this research, the self-healing material under study is an ethylene-co-methacrylic acidbased ionomer partially neutralized with sodium (Surlyn ${ }^{\circledR} 8940$, provided by DuPont ${ }^{\mathrm{TM}}$ Italy). Polymer pellets are dried in vacuum at $60^{\circ} \mathrm{C}$ for $5 \mathrm{~h}$ and square plates $(120 \times 120 \mathrm{~mm})$ of different thicknesses (ranging from 1 to $3 \mathrm{~mm}$ ) are produced by compression moulding at $180^{\circ} \mathrm{C}$. Before testing, specimens are stored in an environmental chamber at $23^{\circ} \mathrm{C}$ and $50 \%$ relative humidity for 1 month to reach stable mechanical properties [8].

A second type of specimen is a multilayer composite, which is made of honeycomb interposed between an ionomer plate and a composite carbon fibre plate (Fig. 1).

The Nomex ${ }^{\circledR}$ (DuPont), an aramidic-based honeycomb, was chosen, being the golden standard in the aerospace industry. Honeycomb's mechanical properties are strictly dependent on the cell geometry. In the present study, an hexagonal structure with a thickness of 6.35 mm, known with the commercial name of HexWeb ${ }^{\mathrm{TM}} \mathrm{HRH}$ 10/3.2/48 (the numbers specify fibre's type/cell diameter $(\mathrm{mm}) /$ density $\left(\mathrm{kg} / \mathrm{m}^{3}\right)$ ] was selected.

Methyl-cyanoacrylate adhesive granted the necessary adhesion between the ionomer and the carbon fibre panel with the interposed honeycomb.

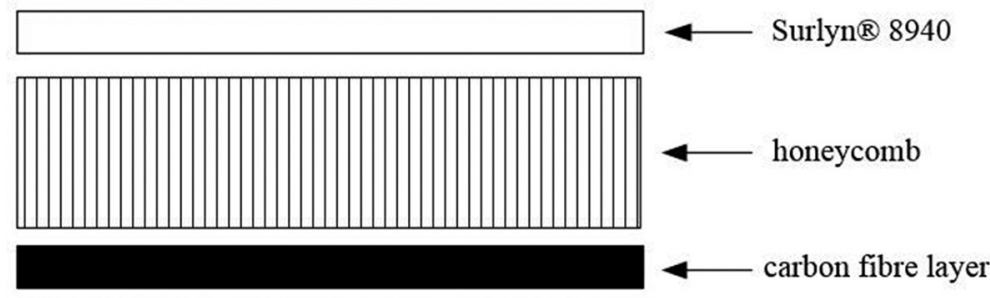

Figure 1: Multilayer panel scheme. 


\subsection{Ballistic tests and healing evaluation}

Ballistic puncture tests were performed in both air and water environment. In the former case, ionomer and multilayer specimens were installed on a steel rigid support with the possibility to set the inclination with respect to the direction of the incoming projectile. In the latter, only the ionomer configuration was tested: the panel was placed as a wall on the entry side of an experimental custom-built aluminium tank, with the ability to withstand forces arising from impact and pressure variations. The tank has a cylindrical shape, with an internal diameter of $92 \mathrm{~mm}$, a length of $366 \mathrm{~mm}$ and an approximate volume of $2.4 \mathrm{~L}$. The specimen was clamped on one base of the cylinder by a square flange with a hole of $92 \mathrm{~mm}$ of diameter.

It was also possible to measure the internal pressure through a transducer installed on the lateral cylindrical wall of the tank, placed $37 \mathrm{~mm}$ from the end.

The internal aluminium filler Explosafe could be placed inside the tank. The filler is made of an aluminium sheet (thickness $0.05 \mathrm{~mm}$ ) manufactured by slitting and expanding the sheet through two mechanical machines to create hexagonal holes [9]. The average density of Explosafe is $30 \mathrm{~kg} / \mathrm{m}^{3}$. During the entire experimental campaign, a cylindrical-shaped block of Explosafe was used. Single layers were rolled to match precisely the internal diameter of the tank, and then inserted in the cylinder (Figs 2-4). The filler was specifically targeted to grant contact with all the tank's internal surfaces.

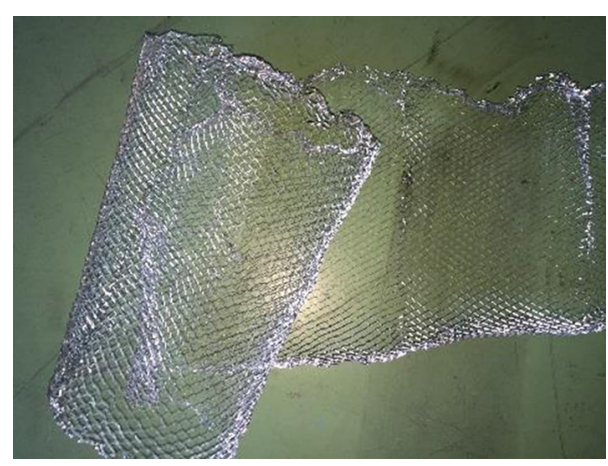

Figure 2: Explosafe filler.

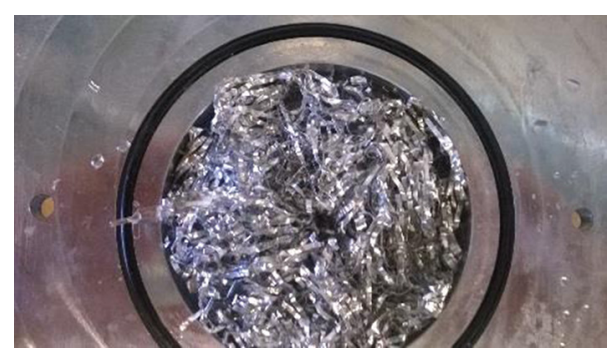

Figure 3: Fuel tank with filler: front view. 


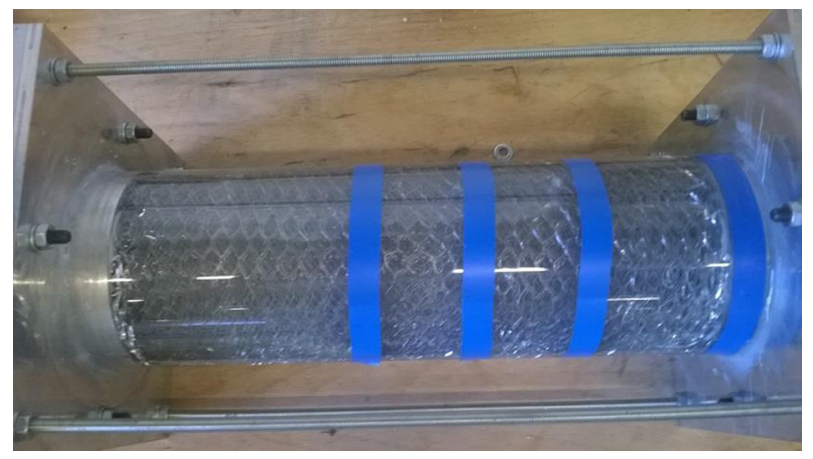

Figure 4: Fuel tank with filler: side view.

Ballistic tests were performed at the LaST laboratory of the Aerospace Science and Technology Department of the Politecnico di Milano. The projectiles used were spherical steelballs with different sizes (up to $16.6 \mathrm{~mm}$ diameter). These were accelerated up to $180 \mathrm{~m} / \mathrm{s}$ with a compressed air device [5]. A high-velocity Phantom v5.1 camera was used to measure the projectile's speed before and after the impact.

After testing all specimens were observed by optical and scanning electron microscope (Hitachi ${ }^{\mathrm{TM}}$ 3000) both in the bullet entrance and exit sides to have visual evidence of hole closures.

To check the healing, leakage tests were carried out using a vacuum pump on one side of the specimen with a pressure difference of 0.9 bars. Air tightness through the hole was tested following vacuum decay. In case of healed hole, no appreciable vacuum decay was detected within a specified time range (10 min), whereas for non-healed samples vacuum decay was observed within 1 min.

\section{RESULTS AND DISCUSSION}

\subsection{Ballistic tests at $180 \mathrm{~m} / \mathrm{s}$ for different configurations}

Globally, 65 ballistic tests have been carried out at $180 \mathrm{~m} / \mathrm{s}$ in four different configurations: ionomer in air, ionomer in contact with water, ionomer in contact with water with Explosafe inside the tank and sandwich in air.

In each configuration, healing was possible if the ratio of the panel thickness (s) and the sphere's diameter (d) was higher than a characteristic number. In other words, for a given bullet diameter healing could be possible in panels thicker than a minimum value. From the healing results, a graph was built with the limit characteristic ratio for every explored condition, each line representing data for every experimental configuration (Fig. 5).

Taking the ionomer in air as a reference, somewhat improved healing performance was noticed in case of the water tank configuration. Healing is probably enhanced by water pressure against the panel, which reduces the overall panel deformation and, possibly, by a positive cooling effect.

Analogous behaviour was not found when the filler Explosafe was introduced in the water filled tank: in this case, ionomer's self-healing behaviour was significantly worse with respect to the reference air case. This negative effect was probably due to the mechanical interaction 


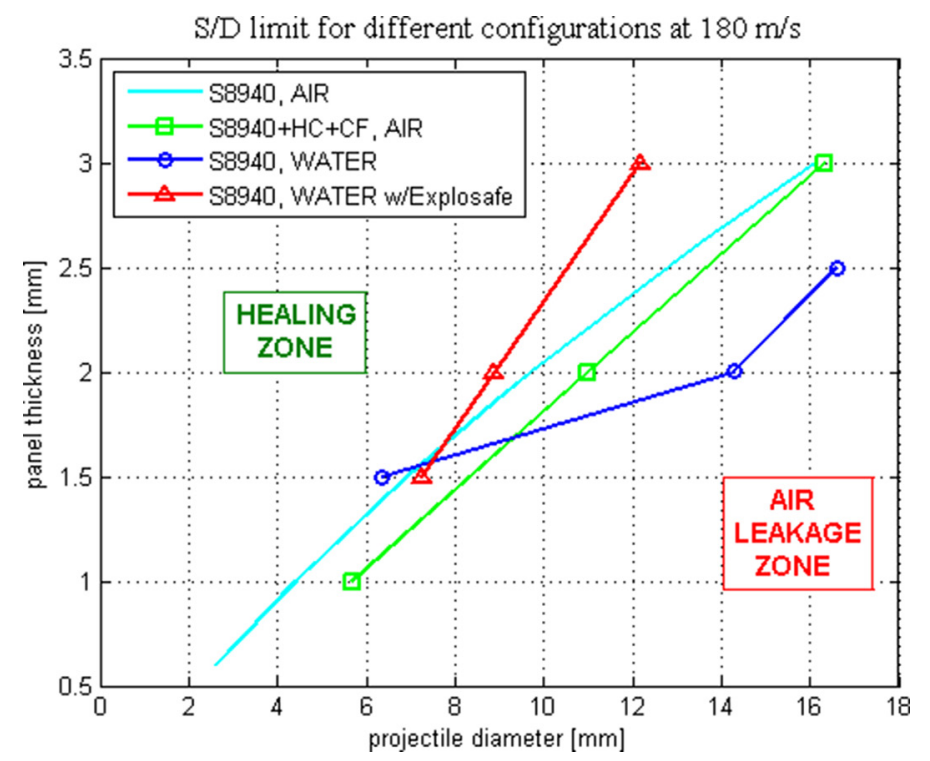

Figure 5: Panel thickness versus projectile diameter.

Table 1: Panel material comparison at equal weight.

\begin{tabular}{lccc}
\hline & Surlyn $^{\circledR} 8940$ & Aluminium (2024-T3) & Steel \\
\hline Density $\left(\mathrm{kg} / \mathrm{m}^{3}\right)$ & 950 & 2800 & 7800 \\
Thickness $(\mathrm{mm})$ & 3 & 1.02 & 0.37 \\
Thickness $(\mathrm{mm})$ & 2 & 0.68 & 0.24 \\
\hline
\end{tabular}

between the panel and the internal filler, which increases the shock wave intensity in proximity of the wet side of the panel; a further possible reason is the cut of the molten ionomer portion during impact deformation, which comes in contact with the sharp hexagonal cells of the aluminium filler. Further studies will explore this interaction.

The multilayer panel has similar performances with respect to the reference case, with small benefits given by the honeycomb support during the initial deformation at impact. Tests performed with $10 \mathrm{~mm}$ diameter steel balls impacting at angles between $45^{\circ}$ and $90^{\circ}$ either on honeycomb multilayers and on plain ionomer did not evidence a clear effect of impact angle on healing capability; provided the impact energy is sufficient for panel perforation, the probability of self-repair remains almost unvaried irrespective of the projectile angle.

It is interesting to observe that, in addition to self-healing feature, a weight reduction may be achieved compared to metal tank design even in terms of perforation resistance. In fact, a 3-mm ionomer panel has a ballistic limit (i.e. the minimum velocity at which a panel is perforated by a projectile) at about $165 \mathrm{~m} / \mathrm{s}$. A panel of $2.37 \mathrm{~mm}$ of 2024-T3 aluminium [10] and one of $0.4 \mathrm{~mm}$ of steel [11] have similar ballistic limit. However, in terms of panel weight, considering its lower density, the Surlyn 8940 solution is convenient with respect to the aluminium alloy and almost equal to the steel one (see Table 1). 


\subsection{Pressure measurements inside the tank in ballistic tests}

Tests with a pressure transducer highlighted the different behaviour between the case with and without Explosafe inside the tank. The test campaign consisted in 15 ballistic impacts ( 8 with the filler). As expected, the first observation is a very strong decrease of sloshing inside the tank when Explosafe is present. Without Explosafe, the projectile impact on the panel and the passage through the water tank caused a visible wave, which was also detected by the pressure sensor. The wave time is in the range of few seconds. On the contrary, with the Explosafe filler, in all the tests a peak wave reduction was observed within $1 \mathrm{~s}$ after the impact (Fig. 6).

With regard to the peak pressure, there is more ambiguity in the tests: in some cases the peak pressure was greatly lower, while in other experiments it was slightly higher. At the moment, the hypothesis is that the interaction between the filler and the ionomeric panel during the deformation stage is the cause of this pressure increase. As a matter of fact, it should be considered that the polymeric wall can deflect extensively, so that it may become in contact with the contained filler during impact. The compression of Explosafe in this phase can result in an additional pressure component, increasing the value of the measured peak pressure. The different rupture modes of the panel are a further complication in the comparison analysis.

\subsection{Holes' partial closure}

In all configurations, even when full healing did not occur, the hole closure was substantial. In more than $90 \%$ of the situations when healing was not attained, the maximum residual hole diameter was about $1 \mathrm{~mm}$ or less. Water spillage occurred at the high peak pressure measured

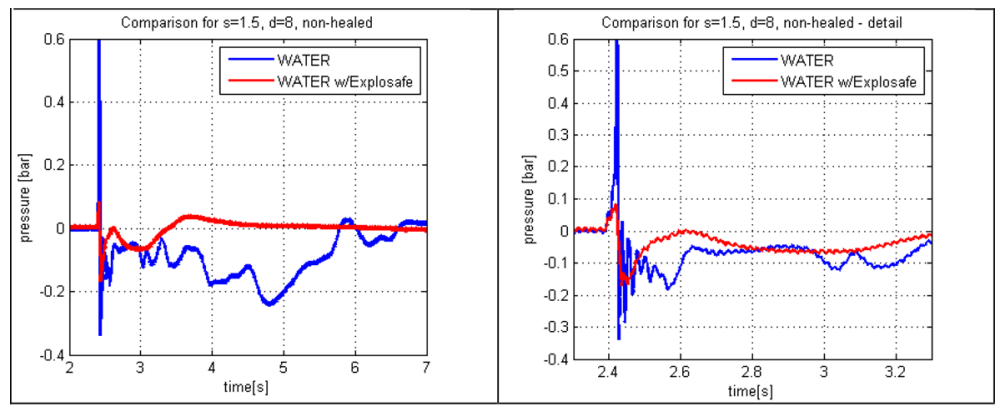

(a)

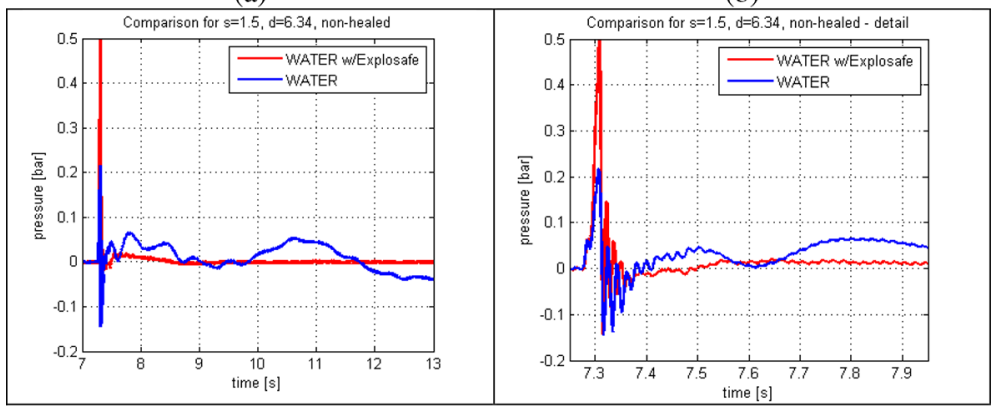

(c)

(d)

Figure 6: Internal tank pressure. 
but, after the first few seconds, the water spillage stopped even if the panel was not fully repaired. It should be noted that the mentioned advantages are not likely to happen in traditional materials like aluminium or steel where the diameter hole is normally equal or larger than the bullet diameter $[10,11]$.

\section{CONCLUSIONS}

Ionomer Surlyn 8940 behaviour under ballistic impact at velocities around $180 \mathrm{~m} / \mathrm{s}$ has demonstrated beneficial effects in containing liquid spillage. The ionomer heals under a wide range of impact conditions. Even in the case of not perfect healing, in the majority of the cases the residual hole due to the spherical projectile passage was one order of magnitude smaller than the cross-section area of the projectile itself. Through this small hole, also thanks to the ionomer chemical features, water passage is very difficult, and in the case of fluid at rest, only a few drops spill out the tank. Results showed that the liquid enhances the healing capabilities. Advantages in terms of weight saving can also be expected from ionomer compared to metal tanks.

The tested integrated solution with Explosafe has shown a complex behaviour; however, a clear evidence of sloshing reduction was always noticed. Peak pressure was not always reduced when the Explosafe filler was used, and thus further investigations should be carried out to identify the real cause of this fact.

A multilayer solution could be adopted, which is able to maintain the self-healing behaviour, when stiffer and stronger walls are required.

\section{REFERENCES}

[1] Bein, D.P., History of fire suppression in aircraft (Chapter 2). Advanced Technology for Fire Suppression in Aircraft, ed. G. Gann, NIST Special Publication 1069, 2007.

[2] Caprile, C., Janszen, G. \& Morandini, M., Study of the effects of a particular fuel tank filler in crash environment. Proceedings of 16th AIDAA National Conference, Palermo, Italy, 2001.

[3] Janszen, G. \& Pernechele, A., Experimental analysis and numerical simulation of a fuel tank filler in crash environment. Proceedings of 3rd International Conference on Fluid Structure Interaction, La Coruña, Spain, 2005.

[4] Francesconi, A., Giacomuzzo, C., Grande, A.M., Mudric, T., Zaccariotto, M., Etemadi, E., Di Landro, L. \& Galvanetto, U., Comparison of self-healing ionomer to aluminium alloy bumpers for protecting spacecraft equipment from space debris impacts. Advances in Space Research, 51, pp. 930-940, 2013. doi: http://dx.doi.org/10.1016/j. asr.2012.10.013

[5] Grande, A.M., Castelnovo, L., Di Landro, L., Giacomuzzo, C., Francesconi, A. \& Rahman, Md.A., Rate dependent self-healing behavior of an ethylene-co-methacrylic acid ionomer under high energy impact conditions. Journal of Applied Polymer Science, 130, pp. 1949-1958, 2013. doi: http://dx.doi.org/10.1002/app.39384

[6] Janszen G., Grande A.M., Bettini P. \& Di Landro L., Integrated solutions for safe fuel tanks. International Journal of Safety and Security Engineering, 4(3), pp. 271-279, 2014. doi: http://dx.doi.org/10.2495/SAFE-V4-N3-271-279

[7] Grande, A.M., Castelnovo, L., Di Landro, L., Sala, G., Giacomuzzo, C. \& Francesconi, A., Multilayer composites with self-healing capability based on an EMAA ionomer. Proceedings of ICCM19, Montreal, Quebec, pp. 2593-2600, 2013. 
[8] Kohzaki, M., Tsujita, Y., Takizawa, A. \& Kinoshita, T., The crystallization and formation of cluster of ethylene ionomer during physical aging. Journal of Applied Polymer Science, 33, pp. 2393-2402, 1987. doi: http://dx.doi.org/10.1002/app.1987.070330710

[9] Szego, A., Premji, K. \& Appleyard, R.D., Evaluation of Explosafe explosion suppression for aircraft fuel tank protection. AFWAL-TR-80-2043.

[10] Loikkanen, M.J., Buyuk, M. \& Kan, C.D., A computational and experimental analysis of ballistic impact to sheet metal aircraft structures, 5th European LS-DYNA Users Conference, 3C-79, 2005.

[11] Dean, J., Dunleavy, C.S., Brownb, P.M. \& Clyne, T.W., Energy absorption during projectile perforation of thin steel plates and the kinetic energy of ejected fragments. International Journal of Impact Engineering, 36, pp. 1250-1258, 2009. doi: http:// dx.doi.org/10.1016/j.ijimpeng.2009.05.002 